\title{
Erosion and deuterium retention of CLF-1 steel exposed to deuterium plasma
}

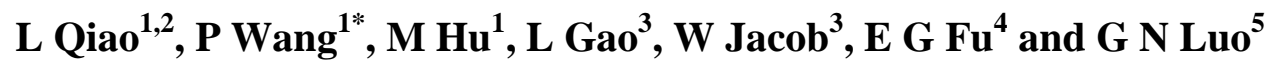 \\ ${ }^{1}$ State Key Laboratory of Solid Lubrication, Lanzhou Institute of Chemical Physics, c, 730000, Lanzhou, PR \\ China \\ ${ }^{2}$ University of Chinese Academy of Sciences, 100049, Beijing, PR China \\ ${ }^{3}$ Max-Planck-Institut für Plasmaphysik, Boltzmannstr. 2, 85748 Garching, Germany \\ ${ }^{4}$ State Key Laboratory of Nuclear Physics and Technology, School of Physics, Peking University, 100871, \\ Beijing, PR China \\ ${ }^{5}$ Fusion Reactor Materials Division, Institute of Plasma Physics, Chinese Academy of Sciences, 230031 Hefei, \\ PR China \\ *Corresponding author: e-mail: pengwang@licp.cas.cn
}

\begin{abstract}
In recent years reduced activation ferritic martensitic (RAFM) steel has been proposed as plasma-facing material (PFM) in remote regions of the first wall. This study reports the erosion and deuterium retention behaviours in CLF-1 steel exposed to deuterium (D) plasma in a linear experimental plasma system as function of incident ion energy and fluence. The incident $\mathrm{D}$ ion energy ranges from $30 \mathrm{to} 180 \mathrm{eV}$ at a flux of $4 \times 10^{21} \mathrm{D} /\left(\mathrm{m}^{2} \mathrm{~s}\right)$ up to a fluence of $10^{25} \mathrm{D} / \mathrm{m}^{2}$. SEM images revealed a clear change of the surface morphology as functions of incident fluence and impinging energy. The mass loss results showed a decrease of the total sputtering yield of CLF-1 steel with increasing incident fluence by up to one order of magnitude. The total sputtering yield of CLF-1 steel after $7.2 \times 10^{24} \mathrm{D} / \mathrm{m}^{2}$ deuterium plasma exposure reduced by a factor of 4 compared with that of pure iron, which can be attributed to the enrichment of $\mathrm{W}$ at the surface due to preferential sputtering of iron and chromium. After D plasma exposure, the total deuterium retention in CLF-1 steel samples measured by TDS decreased with increasing incident fluence and energy, and a clear saturation tendency as function of incident fluence or energy was also observed.
\end{abstract}

Keywords: deuterium retention, erosion, CLF-1 steel, deuterium plasma, sputtering yield

\section{Introduction}

Reduced activation ferritic martensitic (RAFM) steels are being developed for structural applications in future fusion power plants [1-6]. In recent years it has been proposed to use bare RAFM steels as plasma-facing materials (PFMs) in remote regions of the first wall [6-10]. When used as first-wall material in fusion devices, PFMs will be subjected to intense thermal loading and huge fluxes of 
incident particles [1, 6]. To judge if bare RAFM steels can be used as PFMs in the main chamber of DEMO, the interactions of RAFM steels with intensive fluxes of energetic particles and thermal loads need to be investigated.

Many investigations on RAFM steels are focusing on irradiation defects, stability under high temperatures, and changes of mechanical properties [6, 11, 12]. Quite some plasma-wall-interaction (PWI) studies have been performed on EUROFER and F82H, two typical RAFM steel grades. Ashikawa et al. [7] have studied the effects of surface modifications on deuterium retention in $\mathrm{F} 82 \mathrm{H}$ and EUROFER exposed to different fluences ranging from $1 \times 10^{23}$ to $1 \times 10^{25} \mathrm{D} / \mathrm{m}^{2}$ with low-energy deuterium plasmas. It is found that the deuterium retention was $10^{17}-10^{19} \mathrm{D} / \mathrm{m}^{2}$ and the surface concentrations of tungsten increased with the increase of D ion fluence. Similar phenomena have also been observed by Roth et al. [8], Sugiyama et al. [9], and Alimov et al. [10]. Since the sputtering yields of mid-Z elements are significantly higher and sputtering thresholds are lower than those of $\mathrm{W}$ [7-10], there is preferential sputtering of Fe or Cr of RAFM steels after D plasma exposure. Thus W enrichment in the near surface region has been widely reported as a typical feature and this $\mathrm{W}$ enrichment will further affect the erosion behaviour of RAFM steels. All the above reports found also that the total deuterium retention in RAFM steels was lower than in bare $\mathrm{W}$ bulk samples exposed to comparable incident D fluences and ion energies. Moreover, in the ASDEX Upgrade (AUG) [13] and JFT-2M [14] tokamak experiments, researchers have reported the compatibility of RAFM steels with fusion devices, and the experimental campaign did not suffer any particular problem related to the ferromagnetic tiles.

Recently, CLF-1 steel [6] has been developed by SWIP as potential PFM for the Chinese ITER test blanket module. The basic properties of this RAFM steel have also been investigated by quite some groups [15-17]. However, the performance of CLF-1 steel as PFM in terms of erosion and fuel retention has not been studied yet. Related works should be done to allow a confident assessment of the extent to which RAFM steel can be used as PFM in some regions of a fusion device. Therefore, we performed a dedicated set of experiments on the RAFM steels. In this manuscript, using laboratory plasma and ion-beam devices, the fuel retention and erosion were investigated for CLF-1 RAFM steel samples after exposure to energetic deuterium species.

\section{Experimental details}

\subsection{Sample preparation}

All CLF-1 steel samples used in this study were cut from one batch of bulk material to the dimension of $10 \mathrm{~mm} \times 12 \mathrm{~mm} \times 1 \mathrm{~mm}$. Thereafter, the samples were polished with increasingly fine-grained $\mathrm{SiC}$ grinding papers and diamond suspension on the nap cloth to mirror-like finish. Then, to remove water soluble pollutants produced during the mechanical polishing process, samples were polished with deionized water on the nap cloth. Finally the CLF-1 steel samples were cleaned in an ultrasonic bath with acetone for 20 minutes at room temperature. Prior to deuterium implantation the CLF-1 steel 
samples were stress-relieved and degassed by heating at $873 \mathrm{~K}$ in a quartz tube vacuum oven. Prior to annealing treatment, the system was pumped down to a base pressure of less than $1 \times 10^{-5} \mathrm{~Pa}$. Each sample was heated to an annealing temperature of $873 \mathrm{~K}$ with a heating rate of $10 \mathrm{~K} / \mathrm{min}$ and kept for 1 hour at the maximum temperature. The temperature of the sample was calibrated in an independent experiment by a thermocouple fixed to a sample with identical size.

\subsection{Sample characterization}

The surface and cross-sectional microstructures of CLF-1 steel samples were investigated by field emission scanning electron microscope (FESEM, JSM-6701F, JEOL) and high resolution transmission electron microscope (HRTEM, TECNAI G2 S-TWIN F20, FEI; accelerating voltage $200 \mathrm{kV}$ ) equipped with a scanning TEM (STEM) and energy dispersive X-ray spectroscopy (EDS). The ultra-thin slices for HRTEM were prepared using a focused ion beam system (FIB, Helios Nanolab 600i, FEI; Ga ions accelerating voltage $30 \mathrm{kV}$ for preliminary cutting and $5 \mathrm{kV}$ for final milling). The surface roughness for CLF-1 steel was measured on a non-contact white light optical surface profiler interferometry (MicroXAM-800, KLA-Tencor) by using a Gaussian filter.

The mass losses of CLF-1 steel samples were measured by an electronic balance (METTLER TOLEDO, AB135-S) before and after exposure to D plasma. Composition changes of the CLF-1 steel samples before and after exposure to D plasma were determined by Rutherford backscattering spectroscopy (RBS) analysis. A silicon surface barrier detector was placed at the backscattering angle of $160^{\circ}$. The angle of incidence of the projectile ions was $20^{\circ}$ to the surface normal. A beam of $3 \mathrm{MeV}$ ${ }^{4} \mathrm{He}^{+}$with a current of $5 \mathrm{nA}$ was used for measuring the RBS spectra.

\subsection{Deuterium exposure}

The deuterium exposure was performed in the linear experimental plasma system (LEPS) at Lanzhou Institute of Chemical Physics. A basic description of LEPS is given in short: microwave $(2.45 \mathrm{GHz})$ power is introduced into the vacuum chamber through a waveguide terminated by an alumina window. An external magnetic field establishes the electron-cyclotron-resonance condition in the plasma region. During implantation the microwave power is set to $500 \mathrm{~W}$ with a constant $\mathrm{D}_{2}$ gas pressure prior to plasma ignition of $0.3 \mathrm{~Pa}$. The total ion flux impinging on the samples is about $4.0 \times 10^{21} \mathrm{D} /\left(\mathrm{m}^{2} \mathrm{~s}\right)$. It is determined by a Langmuir probe (ESPION). During implantation the sample quickly reaches an elevated temperature in three minutes after starting the plasma. In the following the substrate temperature is maintained at constant temperature by an external open circuit thermostat using cooling water and alcohol. The heating-up time is about $5 \%$ of the total exposure time. The achievable temperatures are between 300 and $500 \mathrm{~K}$ depending on the applied substrate bias voltage. Without cooling, the temperature would increase to much higher values. After stopping the plasma the substrate temperature drops quickly within about three minutes and then slowly approaches room temperature. 
After implantation, the samples were stored in a desiccator in order to avoid air exposure. The ion mass distribution of the impinging ions in LEPS was not yet measured. But because the external plasma operational parameters for LEPS is quite similar as in PlaQ (IPP, Garching) [18], we assume a similar composition of the ion flux. For PlaQ it was shown that the dominant ion species delivered under these conditions is $\mathrm{D}_{3}{ }^{+}[18]$. Together with the plasma potential of about $-15 \mathrm{eV}$, an applied DC bias voltage of $-75 \mathrm{~V}$ will give rise to ion energy of $90 \mathrm{eV}$ and accordingly, $30 \mathrm{eV} / \mathrm{D}$ as impinging energy for the dominant ion species. For PlaQ it was shown that the flux of $\mathrm{D}_{2}^{+}$and $\mathrm{D}^{+}$ions contributes each about $3 \%$ to the total ion flux [18]. These molecular ions give rise to impinging species with higher energies per deuteron (1/2 or the full ion energy per D, respectively). Depending on the presence of impurity species (most probably $\mathrm{O}$ ) in the plasma, the contribution of impurities to sputtering will also increase with increasing energy. Considering the relatively large uncertainty of the sputtering yields due to a possible presence of impurities, it is fair to neglect the contributions of $\mathrm{D}^{+}$ and $\mathrm{D}_{2}^{+}$to sputtering.

\subsection{Thermal desorption spectroscopy}

Thermal desorption spectroscopy (TDS) was used to determine the deuterium release from CLF-1 steel samples after D plasma exposure. In all TDS measurements reported here the maximum temperature was set to $1173 \mathrm{~K}$ with an oven heating rate of $10 \mathrm{~K} / \mathrm{min}$ and the holding time at the maximum temperature was $10 \mathrm{~min}$. To calibrate the temperature response of the samples to the linear oven temperature ramp, an independent experiment was carried out with a CLF-1 steel sample directly attached to a K-type thermocouple during heating. Mass channels between 2 and $44 \mathrm{amu}$ were measured by a quadrupole mass spectrometer (Pfeiffer QME220) as functions of time using multiple ion detection mode (MID). The released fluxes of molecules $\mathrm{HD}$ (mass 3) and $\mathrm{D}_{2}$ (mass 4) were recorded to quantitatively analyze the deuterium retention in CLF-1 steel samples during the temperature ramp [19].

The mass 4 signal was calibrated using a calibrated $\mathrm{D}_{2}$ leak, and the mass 3 signal (HD) was calibrated using the average value of the signals of two calibrated $\mathrm{H}_{2}$ and $\mathrm{D}_{2}$ leaks. The calibration procedure was repeated regularly for $\mathrm{D}_{2}$ and $\mathrm{H}_{2}$ using a calibrated leak (VIC Leak Detection). The total deuterium retention is calculated from the integration of $\mathrm{D}_{2}$ and HD signals from the start of the heating ramp up to the time when the maximum temperature is reached. A background is measured with a sample performing the same heating ramp, but with an empty sample. All TDS data are shown after background subtraction. 


\section{Results and discussion}

\subsection{Surface morphology}

The fluence and energy dependences of the measured surface roughness for CLF-1 steel samples are displayed in figure 1(a). The results show that the surface roughness increased monotonically with increasing incident ion energy and fluence, and no roughness saturation was observed for fluence up to $10^{25} \mathrm{D} / \mathrm{m}^{2}$. Figure 1(b) gives a visual representation of the surface roughness of CLF-1 steel after exposure to D plasma with $180 \mathrm{eV} / \mathrm{D}$ for increasing incident fluence up to $10^{25} \mathrm{D} / \mathrm{m}^{2}$. There was a slight fluctuation in the surface roughness of CLF-1 steel exposure to D plasma with $180 \mathrm{eV} / \mathrm{D}$ for low incident ion fluence, and then an apparently violent fluctuation occurred after exposure to higher fluence.

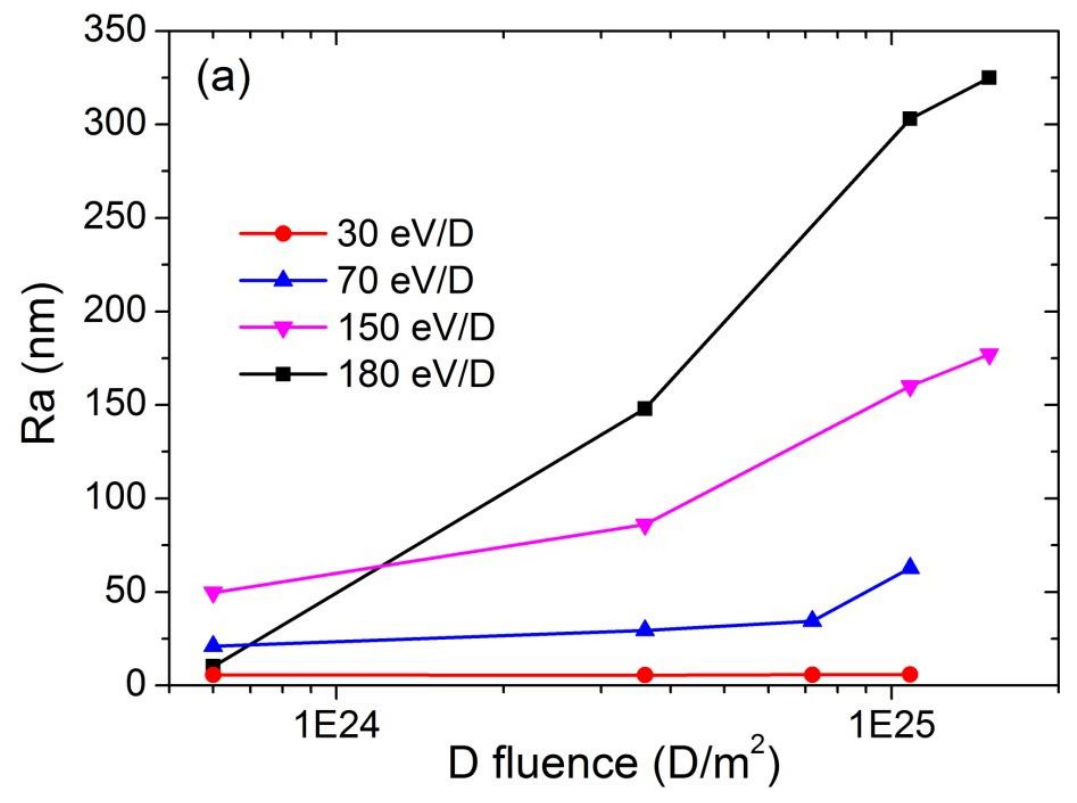




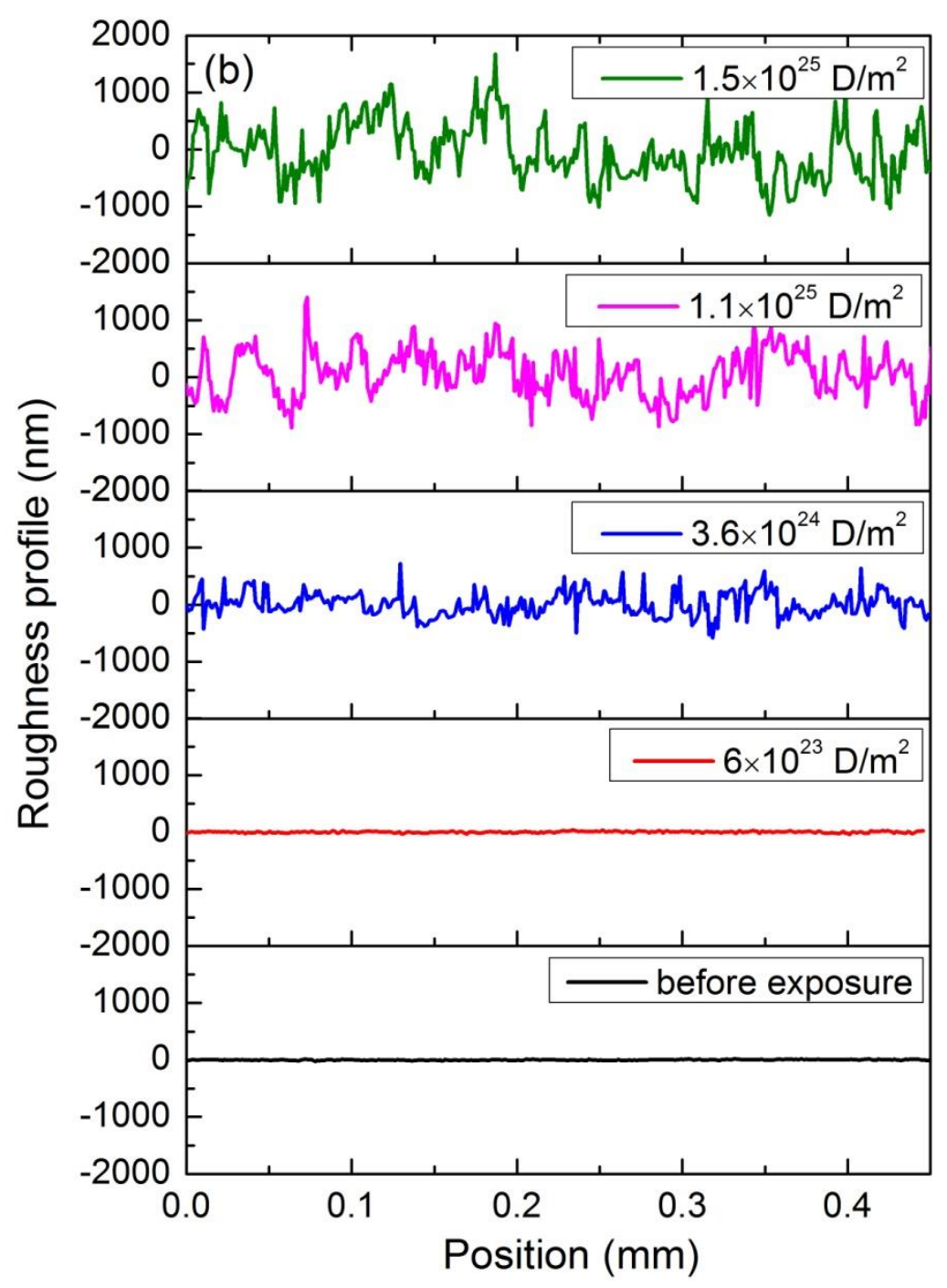

Figure 1. (a) The arithmetical mean deviation of the surface roughness profile (Ra) for CLF-1 steel samples after exposure to D plasma as function of incident deuterium fluence measured for different ion energies. (b) Fluence dependence of surface roughness for CLF-1 steel samples before and after exposure to D plasma with an energy of $180 \mathrm{eV} / \mathrm{D}$. Shown are surface profiles (line scans) after different D exposures. The applied fluences are indicated in the figure.

Surface images of CLF-1 steel samples before and after exposure to D plasma with $150 \mathrm{eV} / \mathrm{D}$ for different deuterium fluences up to $10^{25} \mathrm{D} / \mathrm{m}^{2}$ as examined by SEM are shown in figure 2 . The general microstructure of CLF-1 steel consisted of small grains and some line features due to imperfect polishing as shown in figure 2(a), and these features became less significant after exposure to D plasma. In figure 2(b), after exposure to D plasma for a fluence of $6 \times 10^{23} \mathrm{D} / \mathrm{m}^{2}$, the CLF-1 steel exhibited uneven surface with numerous pits probably due to erosion. Further increasing the incident D fluence, the etching pits were enlarged accompanied by the formation of nano-structure and roughness surface as shown in figure 2(b) and 2(c). More obvious nano-roughness surface modifications ('fuzz'-like) were observed when the incident fluence was higher than $3.6 \times 10^{24} \mathrm{D} / \mathrm{m}^{2}$. 


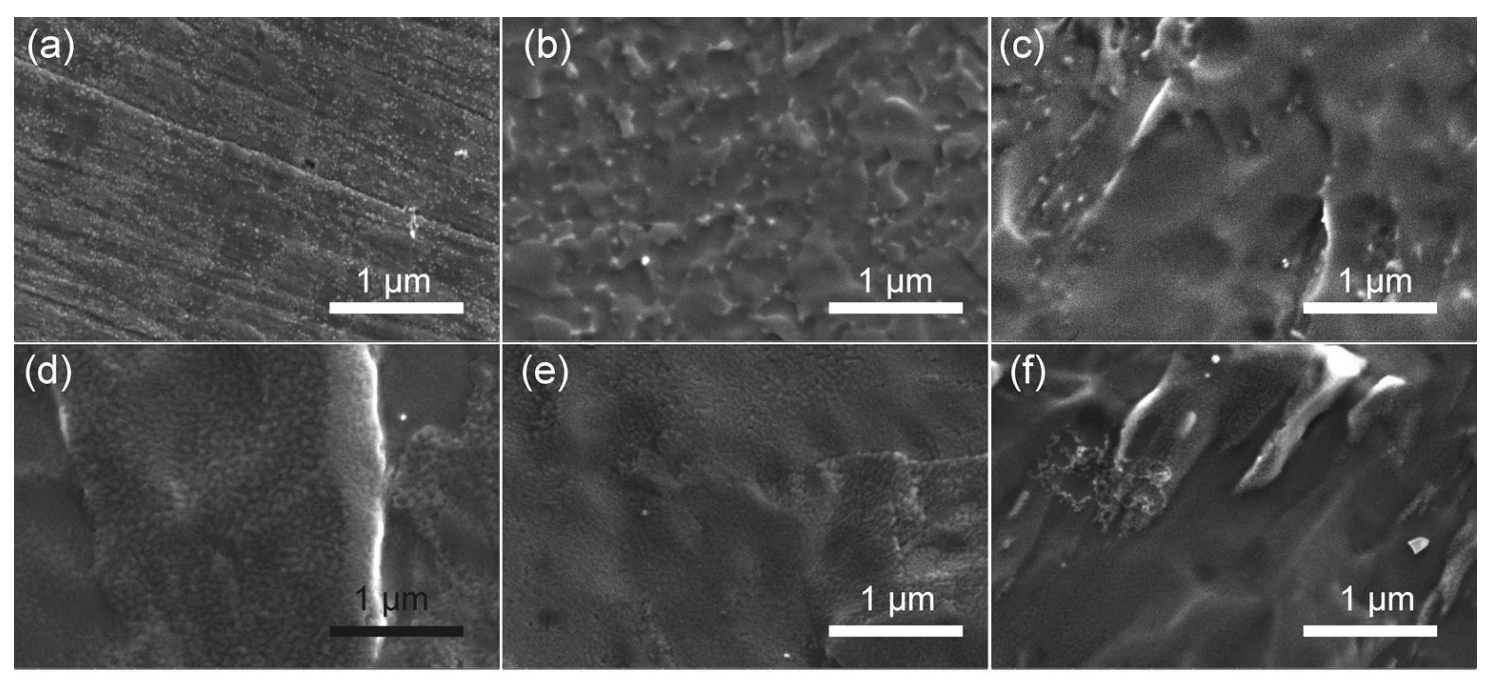

Figure 2. SEM images obtained from the surface of CLF-1 steel samples before (a) and after deuterium exposure with different incident deuterium fluences: (b) $6 \times 10^{23} \mathrm{D} / \mathrm{m}^{2}$, (c) $3.6 \times 10^{24} \mathrm{D} / \mathrm{m}^{2}$, (d) $7.2 \times 10^{24} \mathrm{D} / \mathrm{m}^{2}$, (e) $1.1 \times 10^{25} \mathrm{D} / \mathrm{m}^{2}$, and (f) $1.5 \times 10^{25} \mathrm{D} / \mathrm{m}^{2}$ under a constant energy of $150 \mathrm{eV} / \mathrm{D}$. The straight lines in (a) are remaining groves from the polishing process. Increasing exposure causes a continuous change of the surface morphology.

To investigate the influence of impinging deuterium energy, the surface morphologies of CLF-1 steel samples exposed to D plasma with a constant fluence of $7.2 \times 10^{24} \mathrm{D} / \mathrm{m}^{2}$ are shown in figure 3 for energies ranging from 30 to $180 \mathrm{eV} / \mathrm{D}$. Although $30 \mathrm{eV} / \mathrm{D}$ is below the sputtering threshold of pure iron, a clear sputtering can be found from the surface of CLF-1 steel. This can be attributed to the presence of $\mathrm{D}^{+}$and $\mathrm{D}_{2}{ }^{+}$ions in our deuterium plasma, and at $-90 \mathrm{~V}$ bias the impinging energy of these two ions is $90 \mathrm{eV} / \mathrm{D}$ and $45 \mathrm{eV} / \mathrm{D}$, respectively. Considering that the sputtering threshold of iron is $38 \mathrm{eV} / \mathrm{D}$ [9], the impinging $\mathrm{D}^{+}$and $\mathrm{D}_{2}{ }^{+}$ions can cause sputtering. In addition, plasma impurities, such as e.g. oxygen, can cause additional sputtering. As shown in figure 3, with increasing ion energy, the formation of surface roughness was enhanced accompanied by the increase of pits and 'fuzz' structures. The surface change caused by increasing energy was comparable to that by increasing fluence at $150 \mathrm{eV} / \mathrm{D}$ as shown in figure 2.

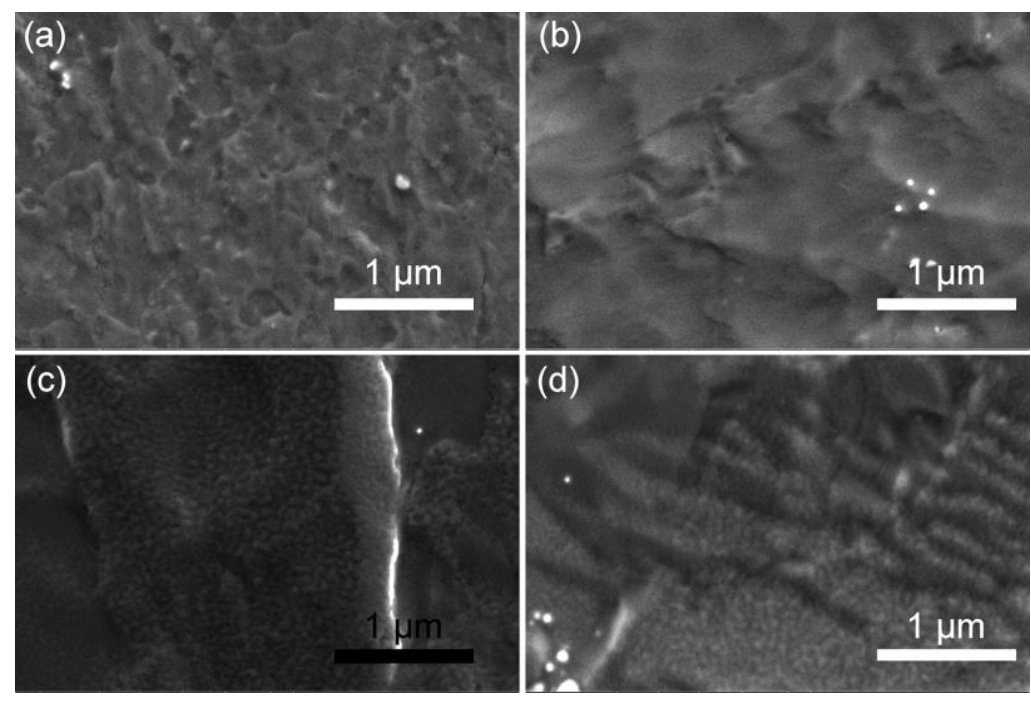


Figure 3. SEM images obtained from the surface of CLF-1 steel samples after deuterium exposure with different impinging energies: (a) $30 \mathrm{eV}$, (b) $70 \mathrm{eV}$, (c) $150 \mathrm{eV}$ and (d) $180 \mathrm{eV}$ per D under a constant fluence of $7.2 \times 10^{24} \mathrm{D} / \mathrm{m}^{2}$. The development of surface morphologies is similar to that shown in figure 2 .

\subsection{Sputtering yields}

Sputtering yields of CLF-1 steel samples obtained from mass loss measurements are shown in figure 4. The total sputtered amounts of CLF-1 steel exposed to D plasma generally increased with increasing impinging energy, which agrees well with the energy dependence of the sputtering of Fe by D [9]. However; the sputtering yields decreased as the incident deuterium fluence increased and saturation was observed for fluences higher than $4 \times 10^{24} \mathrm{D} / \mathrm{m}^{2}$. This decrease is similarly to that observed by Sugiyama et al. [1] after exposing Fe-W binary mixed layer with a low W concentrations of 0.5 and 0.7 at.\%. The $\mathrm{W}$ concentration in the here investigated CLF-1 steel is about $1.5 \%$ [20]. The decrease in sputtering yield with increasing exposure fluence is assumed to be due to $\mathrm{W}$ enrichment, which will be further confirmed by the following TEM and RBS results. In comparison, the sputtering yields of most CLF-1 steel samples are lower than the corresponding theoretical value of pure iron. The total sputtering yield of CLF-1 steel after $7.2 \times 10^{24} \mathrm{D} / \mathrm{m}^{2}$ deuterium plasma exposure is reduced by a factor of 4 compared with that of pure iron, which can be again explained as above that preferential sputtering changes the surface composition, leads to the enrichment of tungsten and thus to a reduction of the sputtering yield. As mentioned above, the surface morphology development and the sputtering yield evolution of CLF-1 steel during exposure to D plasma depend strongly on the surface concentration, i.e., $\mathrm{W}$ enrichment, therefore we will focus on the characterization of the surface in the following.

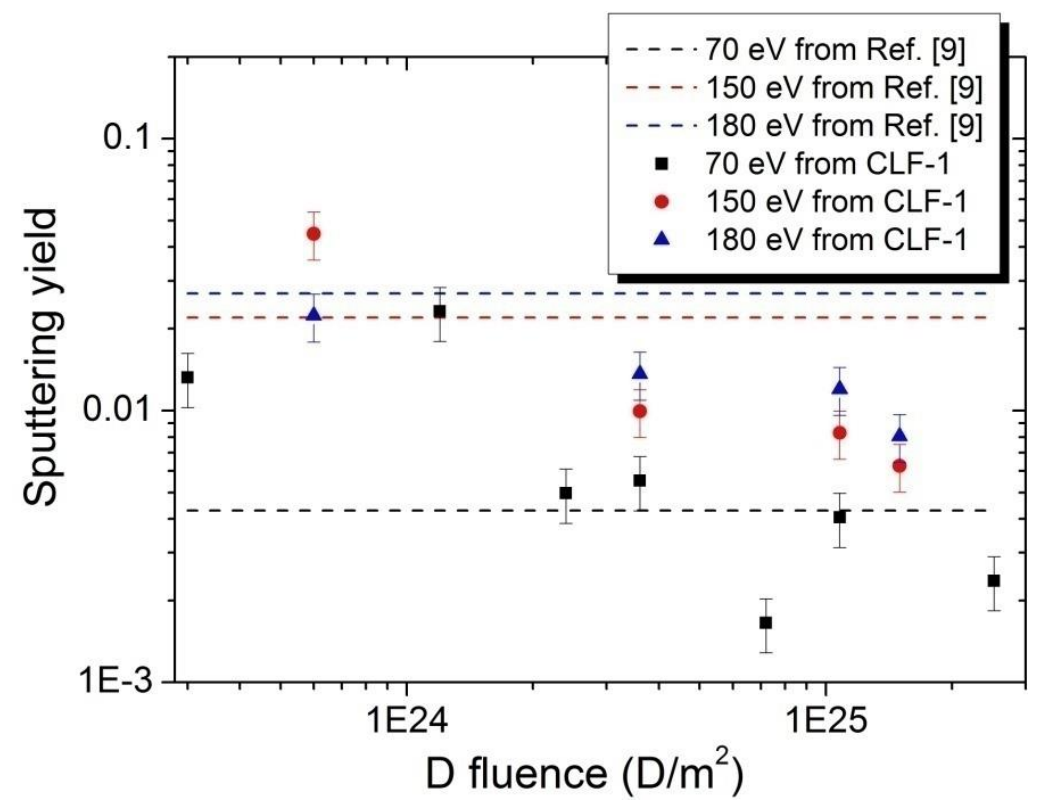

Figure 4. Fluence dependence of the sputtering yields for CLF-1 steel samples at different energies. For comparison, the theoretical sputtering yields of Fe/D as reported by Sugiyama et al. [9] are also shown (dashed lines).

\subsection{Tungsten enrichment}


A cross section of the near surface region of a CLF-1 steel sample after exposure to D plasma ( $150 \mathrm{eV} / \mathrm{D}, 7.2 \times 10^{24} \mathrm{D} / \mathrm{m}^{2}$ ) was investigated by HRTEM. A thin lamella (with an approximate thickness of $50 \mathrm{~nm}$ ) covering a depth range of about $3 \mu \mathrm{m}$ perpendicular to the sample surface was prepared by FIB milling. Prior to FIB milling a protective Pt-C layer is deposited onto the sample surface to protect the surface during FIB milling. This protective layer appears as the granular region in the upper part of the image. HRTEM images of the lamella are shown in figure 5. The obvious martensitic laths in the cross-sectional HRTEM image can be seen in figure 5(a). It shows that the matrix of the sample is in crystalline phase. Around the martensitic lath boundaries some precipitates together with defects such as dislocation cells and sparse dislocation networks in the matrix are observed. All these internal defects and dislocations are typical for the microstructure of CLF-1 steel $[6,16,21]$, and no obvious distortion is observed after exposure.

Figure 5(b) shows an enlarged view of the near surface region. A thin grey region with an approximate thickness of about $20 \mathrm{~nm}$ (also marked in the figure) separates the crystalline bulk from the Pt-C protection layer. We assume that this grey region represents the $\mathrm{W}$ enrichment layer formed during erosion due to preferential sputtering of $\mathrm{Fe}$ and $\mathrm{Cr}$. We measured EDX elemental scans along a cross section perpendicular to the sample surface for this lamella (not shown). However, the Pt EDX line partially overlaps with the $\mathrm{W}$ line such that a small fraction of $\mathrm{W}$ in a large background of $\mathrm{Pt}$ (stemming from the protection layer) cannot be safely distinguished in the EDX line scans. Therefore, a possible enrichment of $\mathrm{W}$ at the surface cannot be proven by EDX. Nevertheless, we found that in the grey region of figure 5(b) the Fe and Cr EDX signals increase from background level to their maximum level and this transition region is about $20 \mathrm{~nm}$ thick.

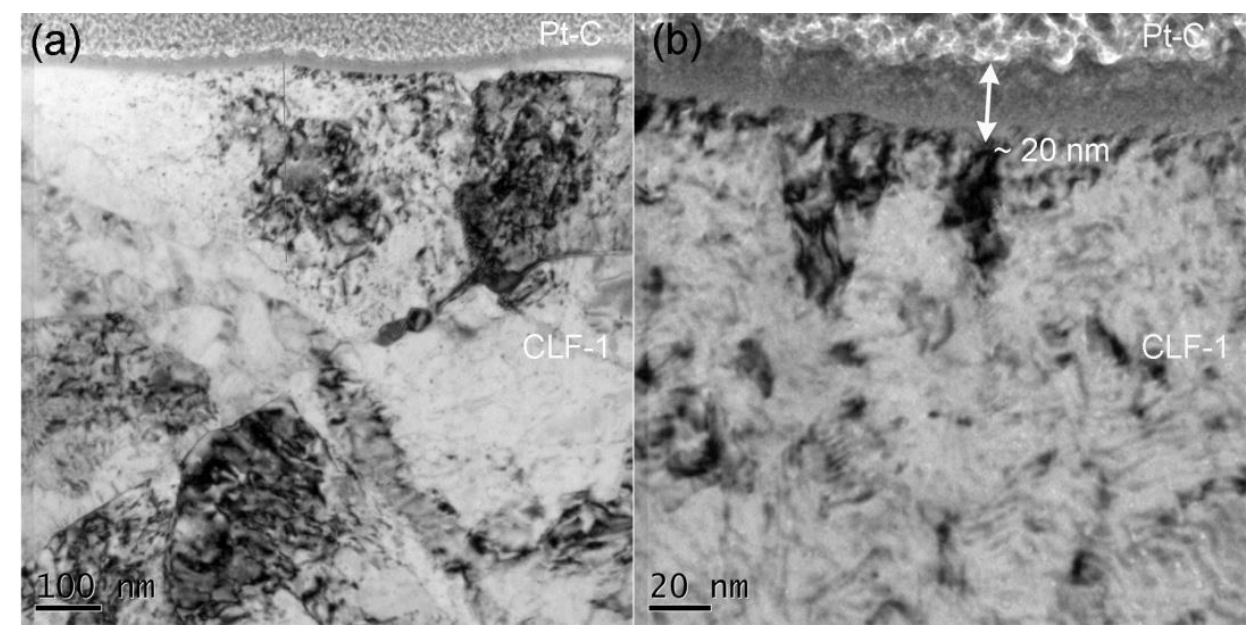

Figure 5. Cross-sectional STEM images of CLF-1 steel after exposure to D plasma with an energy of $150 \mathrm{eV} / \mathrm{D}$ and a fluence of $7.2 \times 10^{24} \mathrm{D} / \mathrm{m}^{2}$. Herein, (a) and (b) are low- and high-magnification images of the region near the top surface, respectively. In figure (b), between the crystalline bulk and the Pt-C protection layer, there is a thin grey region with an approximate thickness of about $20 \mathrm{~nm}$ which is marked with an arrow.

Comparing the RBS spectra of samples before and after exposure to D plasma with $180 \mathrm{eV} / \mathrm{D}$, one can find that the $\mathrm{W}$ concentration on the surface increases after exposure to $\mathrm{D}$ plasma as shown in 
figure 6 , which agrees to the observation shown in figure 5 . The fluence dependence also shows that the $\mathrm{W}$ enrichment at the surface increases with increasing incident deuterium fluence. It also proves the surface enrichment of $\mathrm{W}$ after D exposure. But the enhancement of the W surface peak is not as strong as that reported for F82H steel exposed to comparable incident fluence [7]. This difference can, on the one hand, be explained by the different measurement geometry (in [7] an angle of incidence of $75^{\circ}$ to the surface normal was used which increases the surface sensitivity of the method and enhanced the $\mathrm{W}$ surface peak) or, on the other hand, by the different initial $\mathrm{W}$ concentrations in the used steels (1.5 and $2.0 \mathrm{wt} \% \mathrm{~W}$ in CLF-1 and F82H bulk, respectively).

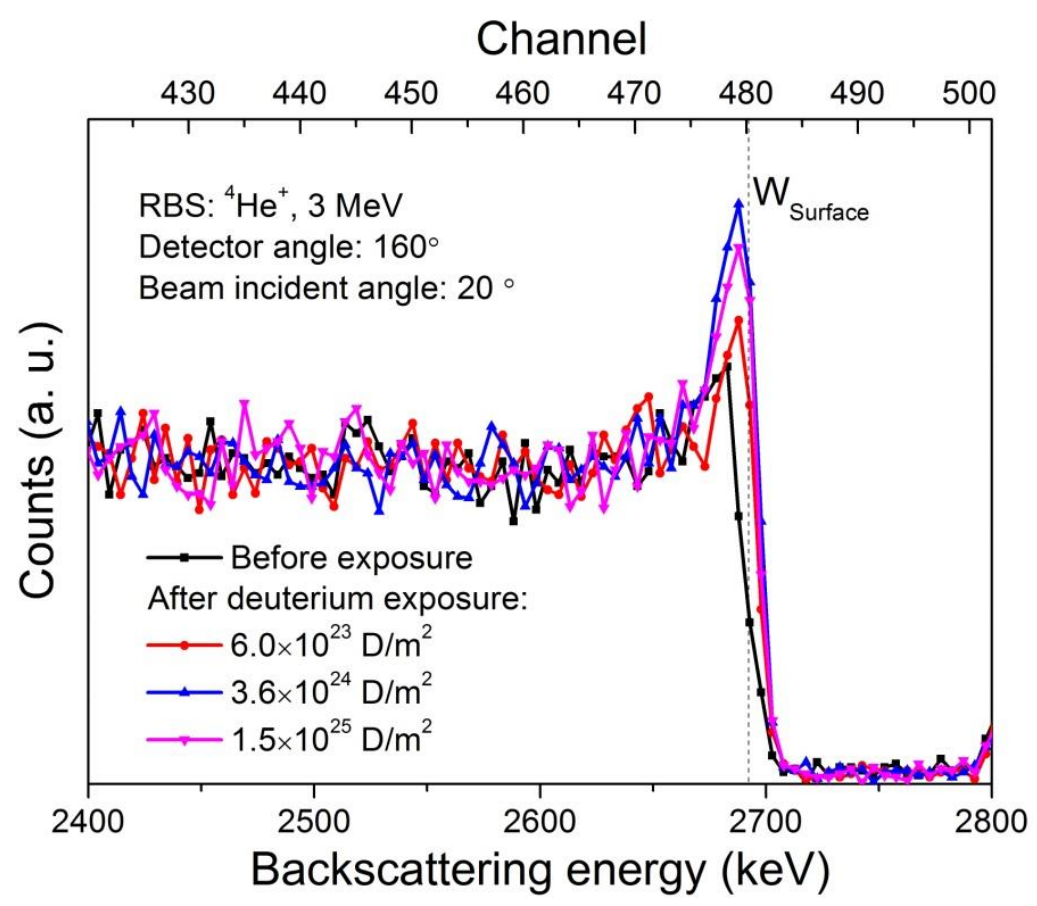

Figure 6. RBS spectra showing backscattering from W from CLF-1 steel samples before and after exposure to D plasma with an energy of $180 \mathrm{eV} / \mathrm{D}$ and different incident fluences. The angle of incidence of the projectile ions was of $20^{\circ}$ to the surface normal. The increase of the $\mathrm{W}$ surface peak (at around $2690 \mathrm{keV}$ ) proves the enrichment of $\mathrm{W}$ at the surface.

\subsection{Deuterium retention}

Thermal desorption spectra of $\mathrm{D}_{2}(4 \mathrm{amu})$ release from CLF-1 steel samples after exposure to $\mathrm{D}$ plasma with a fluence of $7.2 \times 10^{24} \mathrm{D} / \mathrm{m}^{2}$ applying different impinging energies are shown in figure 7(a). The measured HD signals (not shown) have in principle a similar shape as the $\mathrm{D}_{2}$ signals. For all investigated samples the contribution of the HD peak to the total released D amount is at most $30 \%$. Unfortunately, the sample temperature during D exposure increases if the ion energy is increased, such that temperature-induced and ion-energy-induced effects cannot be separated. Therefore, an unambiguous interpretation of the here reported results is presently not possible. D plasma exposure with impinging energy of $30 \mathrm{eV} / \mathrm{D}$ leads to a sample temperature of $330 \mathrm{~K}$. After such exposure deuterium release sets in at about $350 \mathrm{~K}$ and exhibits a high, broad D release peak with the maximum 
at $530 \mathrm{~K}$. Above about $670 \mathrm{~K}$ the peak is followed by a step like shoulder extending up to about $850 \mathrm{~K}$. Above $850 \mathrm{~K}$ the signal decreases again but remains at a non-negligible level up to the maximum temperature. Please observe that the data in figure 7(a) are presented on a logarithmic scale. The signal above $900 \mathrm{~K}$ is very weak and on a linear scale practically invisible. It's contribution to total retention is negligible. Exposure at an energy of $70 \mathrm{eV} / \mathrm{D}$ leads to a sample exposure temperature of $460 \mathrm{~K}$. The corresponding D release spectrum sets in at about $500 \mathrm{~K}$. From $550 \mathrm{~K}$ on it exhibits a broad plateau-like release structure with a comparable intensity as the high temperature shoulder of the sample after $30 \mathrm{eV} / \mathrm{D}$ exposure. This plateau ends in a weak peak at $850 \mathrm{~K}$. For higher temperatures it decreases and finally reaches a level comparable to that of the $30 \mathrm{eV} / \mathrm{D}$ exposure. Exposures at 150 and $180 \mathrm{eV} / \mathrm{D}$ lead both to a sample temperature of $470 \mathrm{~K}$. The release spectra are in principle comparable to that of the $70 \mathrm{eV} / \mathrm{D}$ exposure, only the onset temperatures for $\mathrm{D}$ release are shifted to 550 and $600 \mathrm{~K}$, respectively, and the signal intensities of the plateau levels are slightly different. Interestingly, only the sample with the lowest ion energy/lowest exposure temperature shows a dominant release peak at low temperature while all others only give rise to the broad plateau-like release peak extending up to $\sim 900 \mathrm{~K}$. We assume that the large differences in the measured D release spectra are dominantly cause by the difference in exposure temperatures and not by the differences in impinging energies.

Figure 7(b) shows the total released deuterium amounts calculated from the TDS spectra. The total deuterium retention is calculated from integration $\mathrm{D}_{2}$ and $\mathrm{HD}$ signals as described in Sect. 2.4. After $30 \mathrm{eV} / \mathrm{D}$ exposure the retained amount (about $3 \times 10^{20} \mathrm{D} / \mathrm{m}^{2}$ ) is about one order of magnitude higher than for the other cases. It is clear from a comparison with Fig. 7(a) that this is due to the absence of the low-temperature peak in the latter cases. For this case, the retained amount shows no clear variation with fluence. After exposure with higher energies $(70 \mathrm{eV} / \mathrm{D}$ and higher) the retained $\mathrm{D}$ amounts decrease with increasing D fluence. This puzzling result has already been reported by Ashikawa et al. both for F82H and EUROFER [7]. They suggested that the surface modification, caused by plasma exposure, or possibly a contamination of the surfaces with impurities from the plasma, could be reasons for such counter-intuitive fluence dependence. The data presented in Fig. 7(b) indicate that the total $\mathrm{D}$ retention stabilizes for fluences high than about $5 \times 10^{24} \mathrm{D} / \mathrm{m}^{2}$. The retained amount decrease from about $1 \times 10^{19} \mathrm{D} / \mathrm{m}^{2}$ to about $5 \times 10^{18} \mathrm{D} / \mathrm{m}^{2}$. These values are comparable to those reported by Ashikawa et al. both for F82H and EUROFER [7] for exposure at $450 \mathrm{~K}$. We can, therefore, state that D retention in CLF-1 is comparable to that in F82H and EUROFER. 

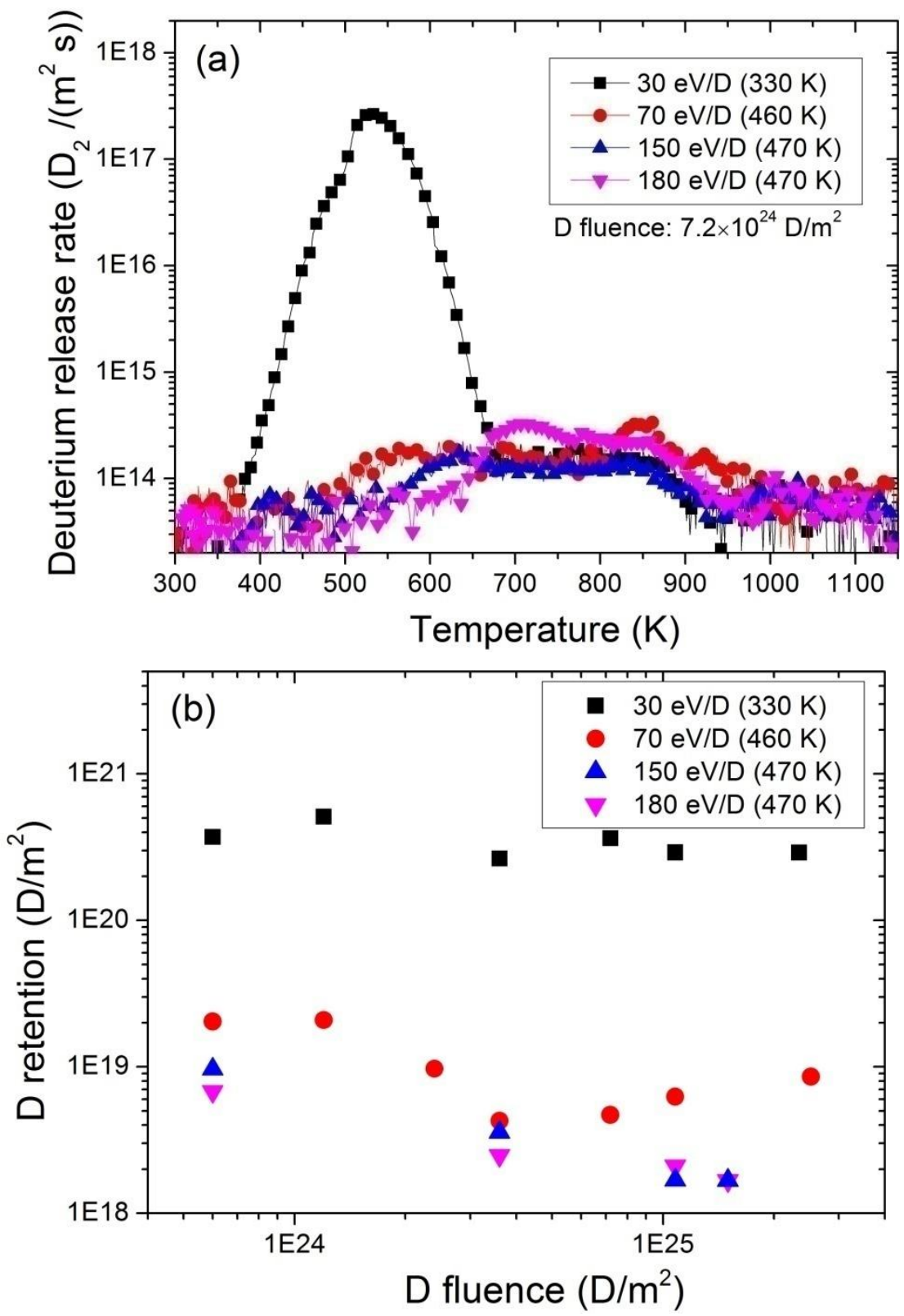

Figure 7. (a) TDS spectra for CLF-1 steel samples after exposure to D plasma with an incident fluence of $7.2 \times 10^{24} \mathrm{D} / \mathrm{m}^{2}$ and different impinging energies. (b) Total deuterium retention for CLF-1 steel after exposure to $\mathrm{D}$ plasma with different impinging energies and incident fluences. Herein, only $\mathrm{D}_{2}$ peaks (4 amu) are shown in 7(a), but both $\mathrm{D}_{2}$ and $\mathrm{HD}$ signals are considered in calculating the total deuterium retention. The sample temperatures during plasma exposure are indicated in the legend.

\section{Summary}

In this study, we investigated the influence of the incident deuterium fluence and impinging energy on erosion and deuterium retention for CLF-1 steel. The results showed a clear change of the surface morphology as a function of energy and fluence (for energies between 30 and $180 \mathrm{eV}$ applying a flux of $4 \times 10^{21} \mathrm{D} /\left(\mathrm{m}^{2} \mathrm{~s}\right)$ up to a fluence of $\left.10^{25} \mathrm{D} / \mathrm{m}^{2}\right)$. The measured sputtering yield decreases with increasing fluence by up to one order of magnitude. RBS analyses revealed that the surface concentration of tungsten increases with increasing fluence due to preferential sputtering of iron and chromium. 
Deuterium release was measured by TDS. Due to the experimental constraint that the exposure temperature and the impinging deuteron energy are correlated, an unambiguous interpretation of the TDS results is not possible. Nevertheless, we assume that the much lower D retention after high energy, high-temperature exposure compared with the low-energy, low-temperature exposure is predominantly due to the higher temperature of the former. The data measured for the high energy, high-temperature exposures are in reasonable agreement with comparable exposures for $\mathrm{F} 82 \mathrm{H}$ and EUROFER and lead us to the conclusion that D retention in these 3 different RAFM steels is comparable.

\section{Acknowledgments}

The authors gratefully acknowledged the financial support from the National Natural Science Foundation of China (Grant No: 11475236). The support from International Thermonuclear Experimental Reactor (ITER) Program with project number of 2015GB121004 from Ministry of Science and Technology of China was also appreciated.

\section{References}

[1] Sugiyama K, Roth J, Alimov V K, et al. 2015 Erosion study of Fe-W binary mixed layer prepared as model system for RAFM steel Journal of Nuclear Materials 463 272-275

[2] Liu P P, Zhan Q, Fu Z Y, et al. 2015 Surface and internal microstructure damage of He-ion-irradiated CLAM steel studied by cross-sectional transmission electron microscopy Journal of Alloys and Compounds 649 859-864

[3] Nishijima D, Baldwin M J, Doerner R P, et al. 2016 Sputtering properties of RAFM steels under high-flux He plasma exposure Nuclear Materials and Energy

[4] Spitsyn A V, Golubeva A V, Bobyr N P, et al. 2014 Retention of deuterium in damaged low-activation steel Rusfer (EK-181) after gas and plasma exposure Journal of Nuclear Materials 455 561-567

[5] Ito T, Yamauchi Y, Hino T, et al. 2011 Deuterium retention in F82H after low energy hydrogen ion irradiation Journal of Nuclear Materials 417 1147-1149

[6] Wang P H, Chen J M, Xu Z Y, et al. 2010 Research and development of reduced activation ferritic/martensitic steel CLF-1 at SWIP 23rd IAEA Fusion Energy Conference, Daejon, Republic of Korea

[7] Ashikawa N, Sugiyama K, Manhard A, et al. 2016 Effects of surface modifications on deuterium retention in F82H and EUROFER exposed to low-energy deuterium plasmas Fusion Engineering and Design 112 236-239

[8] Roth J, Sugiyama K, Alimov V, et al. 2014 EUROFER as wall material: Reduced sputtering yields due to W surface enrichment Journal of Nuclear Materials 454 1-6

[9] Sugiyama K, Schmid K, Jacob W 2016 Sputtering of iron, chromium and tungsten by energetic deuterium ion bombardment Nuclear Materials and Energy 8 1-7 
[10] Alimov V K, Hatano Y, Sugiyama K, et al. 2014 Surface modification and deuterium retention in reduced activation ferritic martensitic steels exposed to low-energy, high flux D plasma and $\mathrm{D}_{2}$ gas Physica Scripta T159 014049

[11] Gaganidze E, Petersen C, Materna-Morris E, et al. 2014 Mechanical properties and TEM examination of RAFM steels irradiated up to 70dpa in BOR-60 Journal of Nuclear Materials 417(1) 93-98

[12] Gaganidze E, Aktaa J. 2013 Assessment of neutron irradiation effects on RAFM steels Fusion Engineering and Design 88(3) 118-128

[13] Zammuto I, Giannone L, Houben A, et al 2015 Long term project in ASDEX upgrade: Implementation of ferritic steel as in vessel wall Fusion Engineering and Design 98 1419-1422

[14] Tsuzuki K, Kimura H, Kawashima H, et al. 2003 High performance tokamak experiments with a ferritic steel wall on JFT-2M Nuclear fusion, $\mathbf{4 3} 1288$

[15] Feng K M, Pan C H, Zhang G S, et al. 2010 Progress on solid breeder TBM at SWIP Fusion Engineering and Design 85 2132-2140

[16] Xia Z X, Zhang C, Lan H, et al. 2010 Influence of smelting processes on precipitation behaviors and mechanical properties of low activation ferrite steels Materials Science and Engineering: A $\mathbf{5 2 8}$ $657-662$

[17] Feng K M, Pan C H, Zhang G S, et al. 2008 Overview of design and R\&D of solid breeder TBM in China Fusion Engineering and Design 83 1149-1156

[18] Manhard A, Schwarz-Selinger T, Jacob W. 2011 Quantification of the deuterium ion fluxes from a plasma source Plasma Sources Science and Technology 20015010

[19] Wang P, Jacob W, Gao L, et al. 2013 Comparing deuterium retention in tungsten films measured by temperature programmed desorption and nuclear reaction analysis Nuclear Instruments and Methods in Physics Research Section B: Beam Interactions with Materials and Atoms 300 54-61

[20] Wang P, Chen J, Fu H, et al. 2013 Effect of $\mathrm{N}$ on the precipitation behaviours of the reduced activation ferritic/martensitic steel CLF-1 after thermal ageing Journal of nuclear materials 442 S9-S12

[21] Wang P, Chen J, et al. 2013 Technical Issues for the Fabrication of a CN-HCCB-TBM Based on RAFM Steel CLF-1 Plasma Science and Technology 15133 